\title{
Coexistence of Autoimmune Hyper- and Hypothyroidism in a Kindred with Reduced Sensitivity to Thyroid Hormone
}

\author{
Yasmine Abdellaoui ${ }^{a}$ Dimitra Magkou ${ }^{b}$ Sofia Bakopoulou ${ }^{b}$ Ramona Zahariab \\ Marie-Laure Raffin-Sanson ${ }^{b, c}$ Laure Cazabat ${ }^{b, c}$ \\ ${ }^{a}$ Department of Internal Medicine, Foch Hospital, Suresnes, France; ${ }^{b}$ Department of Endocrinology and Nutrition, \\ Ambroise Paré Universitary Hospital, Assistance Publique Hôpitaux de Paris, Boulogne, France; ' ${ }^{C}$ A 4340, Université \\ Versailles Saint Quentin en Yvelines, UFR Simone Veil Santé, Montigny le Bretonneux, France
}

\section{What Is Known about This Topic?}

- Both Graves' disease and autoimmune hypothyroidism were described in patients with resistance to thyroid hormone beta (RTH $\beta)$.

- Undiagnosed RTH $\beta$ can lead to misdiagnosis of Graves' disease. The coexistence of these two diseases presents a diagnostic challenge.

\section{What Does This Case Report Add?}

- Autoimmune hypo- and hyperthyroidism may coexist in kindred with RTH $\beta$.

- A rapid increase of suppressed thyroid-stimulating hormone contrasting with persistently elevated thyroid hormones during medical treatment of Graves' disease may indicate RTH $\beta$.

\section{Keywords}

Reduced sensitivity to thyroid hormone - Resistance to thyroid hormone beta . Autoimmune thyroid disease . Graves' disease

\section{Abstract \\ Introduction: Resistance to thyroid hormone beta (RTH $\beta$ ) is a rare disease with an autosomal dominant transmission. Di- agnosis may be challenging especially in patients with hy- per- or hypothyroidism. Case Presentation: A 31-year-old male patient with suppressed thyroid-stimulating hormone (TSH), elevated free thyroxine and free triiodothyronine,}

along with high thyroid receptor antibodies was diagnosed with Graves' disease. Benzylthiouracil was started. One month later, reduced sensitivity to thyroid hormones was suspected because of persistently high thyroid hormone levels contrasting with high TSH level. Molecular analysis highlighted a 10c.1357C > T p.P453S mutation in the thyroid hormone receptor beta gene (THRB). RTH $\beta$ was diagnosed. Several relatives also had RTH $\beta$ (the mother, the young son, and 2 out of 3 siblings). Autoimmune hypothyroidism was present in the mother, whereas 2 out of 3 siblings had asymp-

Y. Abdellaoui and D. Magkou contributed equally to this study.

\section{Karger'?}


tomatic autoimmunity. Discussion/Conclusion:Both Graves' disease and autoimmune hypothyroidism were described in patients with RTH $\beta$. We show here for the first time that autoimmune hypo- and hyperthyroidism may coexist in kindred with RTH $\beta$. Seven previously published cases of Graves' disease and RTH $\beta$ were retrieved and analyzed. Treatments and thyroid hormone level targets are discussed as well as the possible link between RTH $\beta$ and autoimmune thyroid diseases.

(C) 2020 European Thyroid Association Published by S. Karger AG, Basel

\section{Introduction}

Reduced sensitivity to thyroid hormone (TH) [1] is a rare disease with a prevalence of $1 / 40,000$ [2]. It is defined by high levels of free thyroxine (FT4) and free triiodothyronine (FT3) with a normal (but inappropriately elevated) level of thyroid-stimulating hormone (TSH) and encompasses all defects that can interfere with the biological activity of a chemically intact TH [1]. The majority of these patients carry a mutation in the $\beta$ isoform of the thyroid hormone receptor gene (THRB), which has an autosomal dominant transmission, a situation defined in the new nomenclature as resistance to thyroid hormone type beta (RTH $\beta)$ [1]. Goiter, tachycardia, and nervousness are the most common symptoms. The severity of the disease varies among families with THRB mutations [3].
No clear correlation of phenotype with genotype has been found [4]. Autoimmune thyroid diseases (AITD) are relatively common in the general population, frequently showing a familial aggregation. Autoimmune hypothyroidism has been described in families with RTH $\beta$ [5]. Graves' disease (GD) has also been described as isolated cases but no familial aggregation has been reported [6]. We describe herein a family with RTH $\beta$ and AITD manifesting as both hyper- and hypothyroidism.

\section{Case Report/Case Presentation}

A 31-year-old male truck driver presented at the emergency room due to palpitations. Eight years previously, he had undergone radiofrequency ablation of a left septal pre-excitation pathway. He was $180 \mathrm{~cm}$ tall and weighed $80 \mathrm{~kg}$. Atrial fibrillation (AF) was diagnosed as well as a small goiter without any orbital sign. $\mathrm{He}$ received an anti-arrhythmic, a $\beta$-blocker, and anticoagulant treatment. The etiological assessment of atrial fibrillation revealed hyperthyroidism related to GD, with nearly suppressed TSH, elevated FT4 and FT3, along with high thyroid receptor antibodies (TRAb) (Table 1). Thyroid ultrasound revealed a vascular goiter with micronodular dystrophy, and technetium 99m scintigraphy showed a diffuse hyperfixation. Benzylthiouracil (BTU) treatment was started at $200 \mathrm{mg} /$ day. Only 4 weeks later, TSH had normalized, while FT4 and FT3 levels remained elevated (Table 1). Four months later, THs were still supranormal, contrasting with an abnormally high TSH level (Table 1). An assay artifact was dismissed as T4 and T3 antibodies were negative and because repeating the assays using different kits produced the same results. Markers of

Table 1. Evolution of thyroid tests of the index case during and after treatment

\begin{tabular}{ccccll}
\hline & $\begin{array}{l}\text { TSH, mIU/L } \\
(\mathrm{N}: 0.27-4.2)\end{array}$ & $\begin{array}{l}\text { FT4, pmol/L } \\
(\mathrm{N}: 12-22)\end{array}$ & $\begin{array}{l}\text { FT3, pmol/L } \\
(\mathrm{N}: 3.1-6.8)\end{array}$ & $\begin{array}{l}\text { TRAb, IU/L } \\
(\mathrm{N}:<1.8)\end{array}$ & $\begin{array}{l}\text { Treatment } \\
\text { decision: } \\
\text { BTU, mg }\end{array}$ \\
\hline Diagnosis & 0.04 & 54.0 & 22 & 8.8 & 200 \\
1 month & 3.4 & 30.6 & 8.5 & & 200 \\
2 months & 2.29 & 48.4 & 9.7 & 3.52 & 150 \\
3 months & 2.41 & 31.1 & 7.3 & 2.2 & 150 \\
7 months & 4.5 & 29.9 & 8.4 & 0.8 & 150 \\
16 months & 2.5 & 41.7 & 6.1 & 0.75 & 150 \\
20 months & 1.8 & 43.6 & 9.1 & 0.65 & 75 \\
22 months & 1.48 & 33.5 & 8 & & 50 \\
2 years & 1.16 & 42.4 & 5.5 & & 25 \\
30 months & 1.59 & 36.5 & 10.7 & & Stop \\
32 months & 1.35 & 31.8 & 8.6 & $<1$ & 0 \\
3 years & 0.97 & 29.6 & 6.4 & 11 & NMZ 60 mg \\
4 years & 0.99 & 36.6 & 9.7 & 38 & \\
5 years & $<0.01$ & 103 & & & \\
\hline
\end{tabular}

TSH, thyroid-stimulating hormone; FT4, free thyroxine; FT3, free triiodothyronine; TRAb, thyroid receptor antibody; BTU, benzylthiouracil; NMZ, neomercazole. 
TH sensitivity in target tissues (osteocalcin, ferritin, converting enzyme, SHBG, cholesterol) were all normal. Reduced sensitivity to THs was therefore suspected.

The patient reported that several relatives had abnormal thyroid tests and the diagnosis of RTH $\beta$ was confirmed by molecular analysis, which highlighted a $10 \mathrm{c} .1357 \mathrm{C}>\mathrm{T}$ p.P453S mutation in the THRB gene. The patient's familial history was retrieved. Past medical history, TH levels, and genetic sequencing of THRB in the mother and siblings are shown in Figure 1. The P453S THRB mutation was present in the mother (I-1), the youngest brother (II-4), and the son (III-1), but not in a second brother II-3 (Fig. 1). The elder sister (II-1) had a typical RTH $\beta$ hormonal profile and was positive for thyroperoxidase (TPO) antibodies. She reported hyperthyroidism at age 14 with "eye swelling," treated over 3 years with BTU. No biochemical data from that period was available. She refused genetic testing. Autoimmunity was also found in the brother (II-3), who had a normal THRB sequence. The youngest brother (II-4) was diagnosed with RTH $\beta$ without thyroid autoimmunity.

The patient's mother (I-1) had been diagnosed with autoimmune hypothyroidism at 40 years of age. At the time of diagnosis, TSH was measured at $11.4 \mathrm{mIU} / \mathrm{L}(0.35-4.9)$, anti-TPO and antithyroglobulin antibodies were both positive. A thyroid ultrasound emphasized thyroiditis. Under levothyroxine (LT4) at 100 then $150 \mu \mathrm{g} /$ day, her TSH remained high, between 8.95 and $12 \mathrm{mIU} / \mathrm{L}$, while her FT4 level increased to $33 \mathrm{pmol} / \mathrm{L}(9-19)$ and T3 to 6.15 $\mathrm{pmol} / \mathrm{L}$ (2.6-5.53). A TRAb test was negative. LT4 was discontinued by the patient 1 year later because of poor clinical tolerance. At the time of genetic testing, in the absence of treatment, TSH had reached $39 \mathrm{mIU} / \mathrm{L}, \mathrm{FT} 4$ was normal, and FT3 levels were slightly elevated (Fig. 1).
In the absence of prior data for $\mathrm{TH}$ values in the propositus, the therapeutic objective was to achieve levels of FT3 and FT4 that were close to those of the youngest brother (II-4), that is, FT4 between 30 and $40 \mathrm{pmol} / \mathrm{L}$ and FT3 between 7 and $12 \mathrm{pmol} / \mathrm{L}$. Tests for TRAb became negative after 6 months. BTU was stopped after two and a half years (Table 1). Arrhythmia persisted despite external electric shock and a second radiofrequency ablation. The patient remained in remission for more than 2 years and then presented with a clinical relapse of GD with orbitopathy, confirmed by suppressed TSH and very high TH and TRAb levels. Treatment was resumed, with the same therapeutic targets. With regard to the mother, it was decided, after informing the patient in detail, to reintroduce LT4 treatment at very progressive doses, aimed at maintaining TSH levels slightly above the normal range $(5-10 \mathrm{mmol} / \mathrm{L})$ and FT 4 between 25 and $30 \mathrm{pmol} / \mathrm{L}$.

\section{Discussion}

The association of RTH $\beta$ and GD is rare and difficult to diagnose. At presentation, the index patient had typical hyperthyroidism due to GD with suppressed TSH, as expected in the presence of high TH levels, confirming that TSH secretion remains suppressible in RTH $\beta$ if exposed to sufficient TH. The usual evolution of thyroid tests under medical treatment of GD consists in prior normalization of THs, whereas TSH usually remains low for a few weeks or months. In the present case, normal TSH before
Fig. 1. Pedigree of the family and results of thyroid function tests. The arrow indicates the index case. Thyroid function test results are presented under each symbol representing a family member. Abnormal values are in bold. Symbols filled in black on the left indicate subjects with thyroid hormone resistance beta. Symbols filled in white on the left indicate subjects with no thyroid hormone resistance. Symbols hatched on the right indicate subjects with autoimmune hypothyroidism (AH). Symbols checkered on the right indicate subjects with Graves' disease (GD). Symbols filled in white on the right indicate subjects with no thyroid autoimmunity (TAI). Symbols filled in gray indicate the individual was not tested for thyroid function. $T H R B$, thyroid hormone receptor beta; FT3, free triiodothyronine; FT4, free thyroxine; TSH, thyrotropin; TG, thyroglobulin; TPO, thyroperoxidase; Ab, autoantibodies; TRAb, TSH receptor antibodies; wt, wild-type sequence; $\mathrm{N}$, normal range; nd, not done. ${ }^{*}$ II- 1 was tested at 37 years of age, after resolution of a possible GD treated between 14 and 16 years of age.

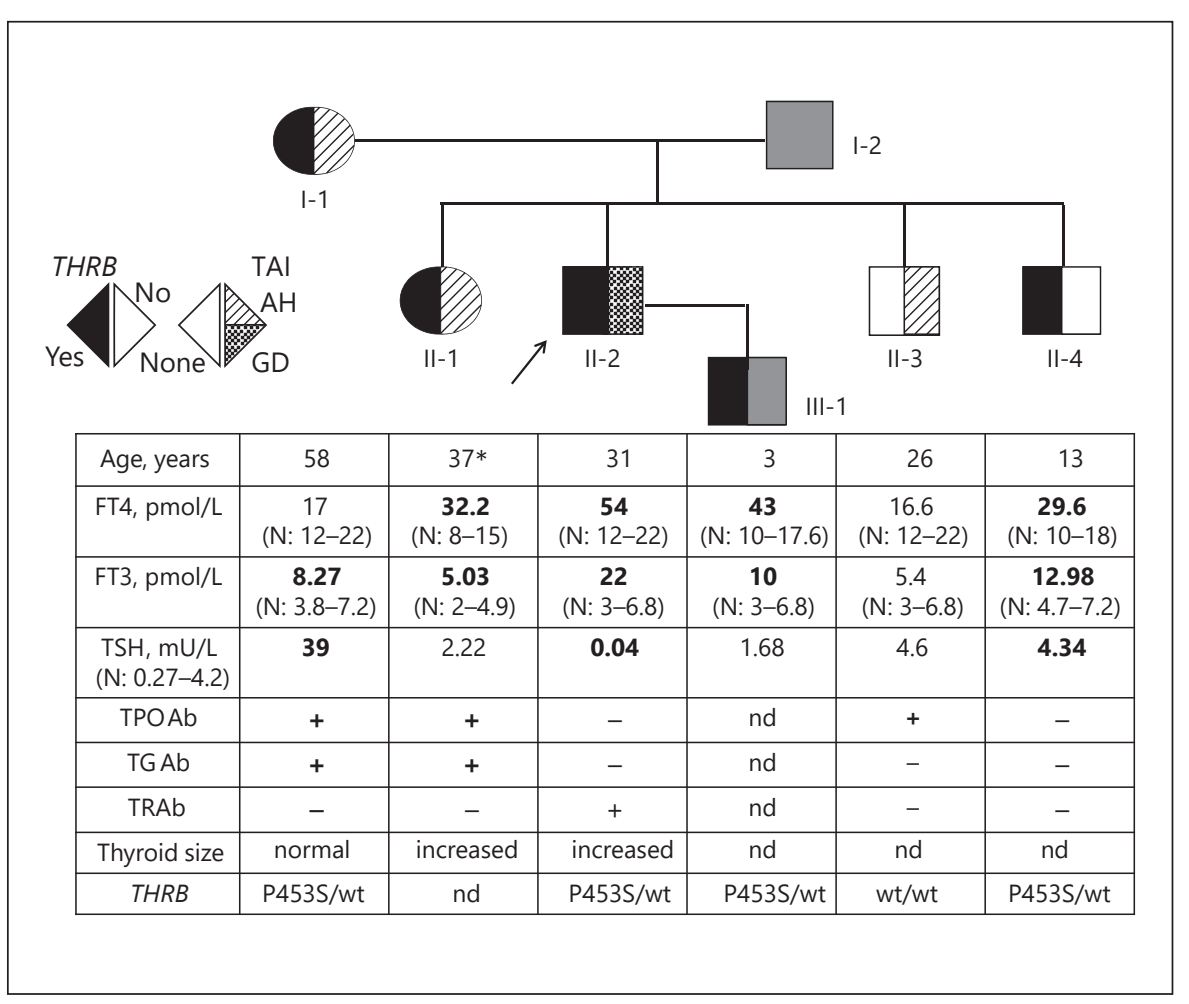

Familial AITD and Reduced Sensitivity to Thyroid Hormone 
Table 2. Published cases with Graves' disease and thyroid hormone resistance beta

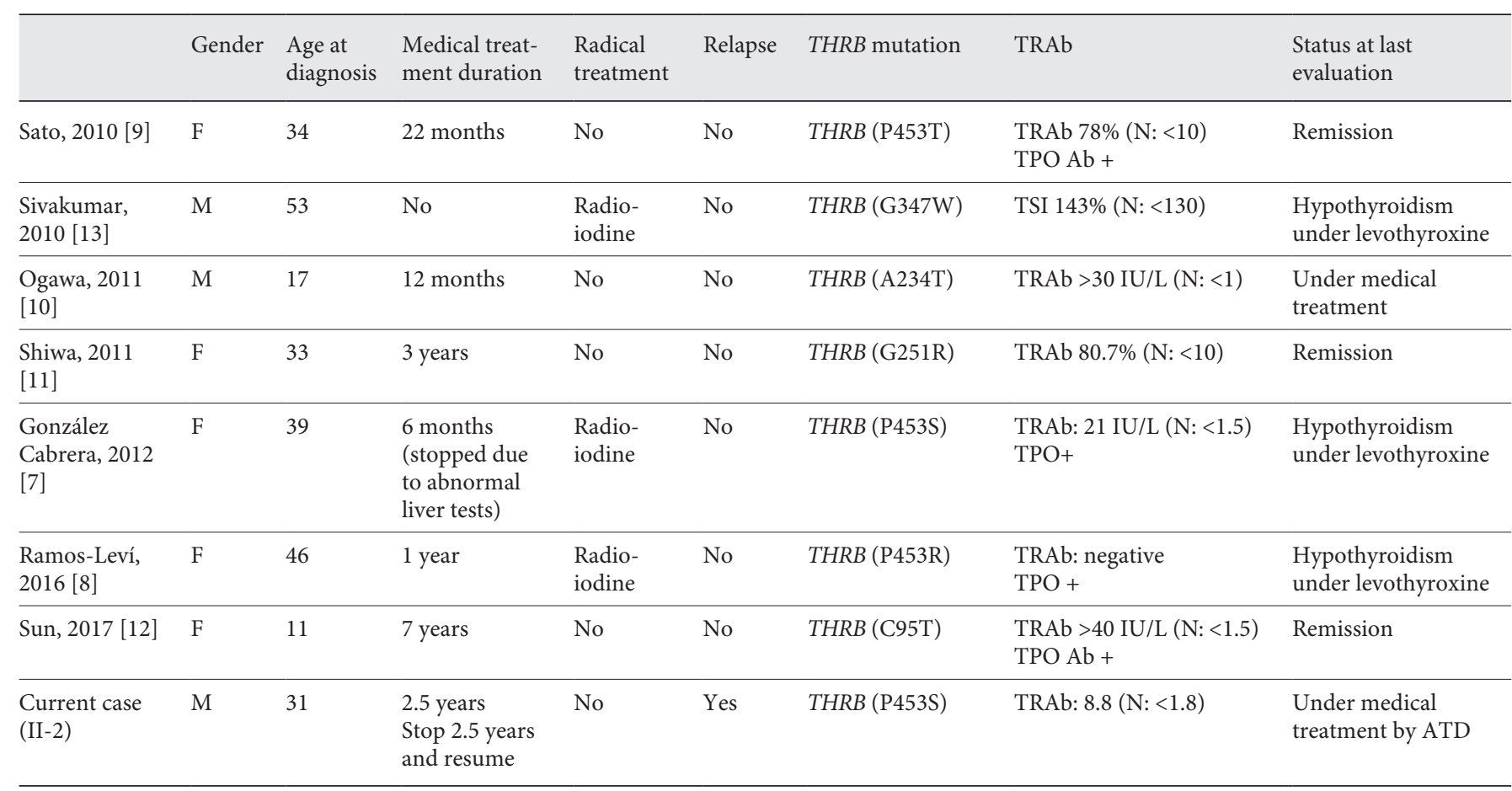

NA, not available; ATD, antithyroid drug; THRB, thyroid hormone receptor beta gene; TRAb, TSH autoantibodies; TPO Ab, anti-thyroid peroxidase autoantibodies.

complete FT4 and FT3 control prompted the endocrinologist to search for an assay artefact, then for RTH $\beta$. Treatment of GD in the context of RTH $\beta$ is difficult. At present, the optimal therapy is unknown. Medical treatment with antithyroid drugs is the first choice, but determining the therapeutic target range for $\mathrm{TH}$ may be difficult when the level of TH before the onset of GD is unknown, as was the case in this patient. Attempts to normalize TH level with antithyroid drugs would likely lead to hormonal failure in peripheral hormone-resistant tissues. It was thus decided to take the FT3 and FT4 levels of his younger brother, with RTH $\beta$ and no thyroid autoimmunity, as a reference and target. Although the same mutation may lead to slightly different thyroid profiles within the same family, this approximation has proven helpful for the patient who has reported clinical wellbeing. Radioiodine therapy or surgery are not usually used as a first therapeutic choice for GD in RTH $\beta$ patients, since they lead to hypothyroidism, while appropriate hormone replacement therapy is also difficult to achieve in these patients. The patient's mother, with RTH $\beta$ and autoimmune thyroiditis, illustrates this difficulty. Although true hypothyroidism is very likely because of the significant elevation of TSH, substitution was not tolerated, probably because of the use of an excessive dose of TH in an attempt to normalize TSH. RTH $\beta$ diagnosis in this patient led to prescription of lower L-thyroxin substitution and an adapted TSH target.

Seven cases of GD in patients with RTH $\beta$ have been published to date, and data from these reports are summarized in Table 2. In three cases, GD was diagnosed in patients with known RTH $\beta$ [7-9]. In 4 cases, RTH $\beta$ was discovered after several years of medical treatment for GD [10-12] or because of the difficulty in equilibrating medical treatment of iatrogenic hypothyroidism after radioiodine treatment [13]. This is the first time, to our knowledge, that RTH $\beta$ was suspected soon after initiating the medical treatment of GD. Among the seven patients with GD in a context of $\mathrm{RTH} \beta$, three received radical treatment with radioiodine, all resulting in hypothyroidism, whereas 4 obtained remission with medical treatment only. Patients with RTH $\beta$ and hypothyroidism require TH supplementation at doses much higher than the usual replacement doses. Reaching thyroid equilibrium in these patients may be difficult to achieve [13]. Interestingly, the mother reported poor clinical tolerance of LT4 substitution in spite of frank hypothyroidism. She described rapid heart rate and palpitations as LT4 was in- 
creased. Two assumptions can be made here. Since RTH $\beta$ was not yet diagnosed when the treatment was initiated, we hypothesized that she had been given too much LT4, in an attempt to normalize TSH, whereas basal TSH is mildly elevated in this RTH $\beta$ family. She would have experienced clinical hyperthyroidism due to excessive LT4 stimulation in TR-a-dependent tissues. Alternatively, unpleasant symptoms frequently present in patients with RTH $\beta$ (tachycardia, palpitation, hyperactivity) due to TR- $\alpha$ excessive stimulation could have been relieved when the patient developed hypothyroidism. Introducing LT4 substitution would have induced a relapse of these symptoms. At the same time, we must assume that in this patient, the lack of TH in tissues in which THRB is predominantly expressed had few noticeable consequences, as is sometimes observed in patients diagnosed with frank biological hypothyroidism in the absence of obvious clinical symptoms.

This family is remarkable in showing the aggregation of thyroid autoimmune diseases, including at least one patient with GD, as the diagnosis could not be firmly established in the sister (II-1). Barkoff et al [14] searched for a possible link between RTH $\beta$ and AITD in 130 families (330 subjects) with RTH $\beta$, confirmed by the presence of $T H R B$ gene mutations, and in 92 unaffected first-degree relatives. They concluded that patients with RTH $\beta$ have an increased likelihood of AITD (defined by the presence of anti-TPO and/or anti-thyroglobulin antibodies) compared to healthy first-degree relatives.

The reason for this link is unknown. It has been proposed that chronic TSH stimulation in RTH $\beta$ could activate intrathyroidal lymphocytes [15] leading to autoimmune hypothyroidism. A parallel decrease of serum TSH and titers of antithyroid autoantibodies is observed in patients with hypothyroidism treated with levothyroxine, as well as coincidence of GD and thyrotropic adenomas [16]. Stimulation of autoimmunity by $\mathrm{TH}-\alpha$ receptors present in epithelial cells of the thymus has also been proposed [17], but only autoimmunity against thyroid has been linked to RTH $\beta$. Thus, the exact nature of the association of RTH $\beta$ and AITD remains to be further investigated.

\section{Conclusion}

The concurrent presence of RTH $\beta$ and AITD in a patient can render both the diagnosis and treatment challenging. TSH is suppressed in GD, even in a context of $\mathrm{RTH} \beta$. When RTH $\beta$ is undiagnosed, a rapid increase in
TSH after the beginning of the treatment of GD contrasting with persistently high $\mathrm{TH}$ levels should raise the diagnosis.

For GD in a setting of RTH $\beta$, medical treatment should be the first line of therapy, the goal of treatment being to obtain TH levels close to those observed before the onset of GD. In the absence of any prior reference, the TH levels of relatives with RTH $\beta$ but unaffected by AITD can be used as a target.

\section{Acknowledgements}

We thank the patients and the staff of the Endocrinology and Nutrition Department of Ambroise Paré Hospital for their participation in this study and Dr. Tatiana Deneuville and Dr. Elodie Le Marois for helpful discussions.

\section{Statement of Ethics}

Patients provided written informed consent for the anonymous publication of their medical data.

\section{Disclosure Statement}

The authors have no conflicts of interest to declare.

\section{Funding Sources}

None.

\section{Author Contributions}

Y. Abdellaoui, S. Bakopoulou, R. Zaharia, and L. Cazabat cared for the patient and collected the medical data. Y. Abdellaoui, D. Magkou, and M.L. Raffin-Sanson wrote the manuscript. All authors approved the manuscript.

References

1 Refetoff S, Bassett JH, Beck-Peccoz P, Bernal J, Brent G, Chatterjee K, De Groot LJ, Dumitrescu AM, Jameson JL, Kopp PA, Murata Y, Persani L, Samarut J, Weiss RE, Williams GR, Yen PM. Classification and proposed nomenclature for inherited defects of thyroid hormone action, cell transport, and metabolism. Eur Thyroid J. 2014 Mar;3(1):7-9.

2 Dumitrescu AM, Refetoff S. The syndromes of reduced sensitivity to thyroid hormone. Biochim Biophys Acta. 2013 Jul;1830(7): 3987-4003. 
3 Refetoff S, Weiss RE, Usala SJ. The syndromes of resistance to thyroid hormone. Endocr Rev. 1993 Jun;14(3):348-99.

4 Beck-Peccoz P, Chatterjee VK. The variable clinical phenotype in thyroid hormone resistance syndrome. Thyroid. 1994;4(2):225-32.

5 Aksoy DY, Gurlek A, Ringkananont U, Weiss RE, Refetoff S. Resistance to thyroid hormone associated with autoimmune thyroid disease in a Turkish family. J Endocrinol Invest. 2005 Apr;28(4):379-83.

6 Smith TJ, Hegedüs L. Graves' Disease. N Engl J Med. 2016 Oct 20;375(16):1552-65.

7 González Cabrera N, Kalic AK, Antón Miguel MÁ, Sierra Polo P, Vicente Vicente MÁ. Hyperthyroidism due to Graves-Basedow disease in a woman refractory to thyroid hormones. Endocrinol Nutr. 2012 Dec;59(10): 609-11.

8 Ramos-Leví AM, Moreno JC, Álvarez-Escolá C, Lacámara N, Montañez MC. Coexistence of thyroid hormone resistance syndrome, pituitary adenoma and Graves' disease. Endocrinol Nutr. 2016 Mar;63(3):139-41.
9 Sato H. Clinical features of primary hyperthyroidism caused by Graves' disease admixed with resistance to thyroid hormone (P453T). Endocr J. 2010;57(8):687-92.

10 Ogawa K, Yoshida M, Hayashi Y, Murata Y, Miyata M, Oiso Y. A rare case of resistance to thyroid hormone coexisting with Graves' disease. Endocrine. 2011 Oct;40(2):318-9.

11 Shiwa T, Oki K, Awaya T, Nakanishi S, Yamane K. Resistance to thyroid hormone accompanied by Graves' disease. Intern Med. 2011;50(18):1977-80.

12 Sun H, Xu S, Xie S, Cao W, Chen G, Di H, et al. Graves' disease coexisting with resistance to thyroid hormone: a rare case. Clin Case Rep. 2017 Dec;6(2):337-41.

13 Sivakumar T, Chaidarun S. Resistance to thyroid hormone in a patient with coexisting Graves' disease. Thyroid. 2010 Feb;20(2): 213-6.
14 Barkoff MS, Kocherginsky M, Anselmo J, Weiss RE, Refetoff S. Autoimmunity in patients with resistance to thyroid hormone. J Clin Endocrinol Metab. 2010 Jul;95(7):3189_ 93.

15 Gavin C, Meggison H, Ooi TC. Proposing a causal link between thyroid hormone resistance and primary autoimmune hypothyroidism. Med Hypotheses. 2008;70(5):10248.

16 Kamoun M, d'Herbomez M, Lemaire C, Fayard A, Desailloud R, Huglo D, et al. Coexistence of thyroid-stimulating hormone-secreting pituitary adenoma and graves' hyperthyroidism. Eur Thyroid J. 2014 Mar;3(1):60-4.

17 Villa-Verde DM, Defresne MP, Vannier-dosSantos MA, Dussault JH, Boniver J, Savino W. Identification of nuclear triiodothyronine receptors in the thymic epithelium. Endocrinology. 1992 Sep;131(3):1313-20. 